\title{
April 2017 Imaging Case of the Month
}

\section{Michael B. Gotway, MD and John K. Sweeney, MD}

\author{
Department of Radiology \\ Mayo Clinic Arizona \\ Scottsdale, Arizona USA
}

Clinical History: An 86-year-old man with a previous history of transcatheter aortic valve implantation 1 year earlier, coronary artery disease status-post coronary artery bypass grafting surgery 12 years earlier, atrial fibrillation on warfarin, and pacemaker placement 8 years earlier presented with altered mental status.

The patient's white blood cell count was borderline elevated at $10.3 \times 10^{3} / \mathrm{mcl}$ (normal, $4.8-10.8 \times 10^{3} / \mathrm{mcl}$ ) and hyponatremia was noted (serum sodium $=129 \mathrm{mEq} / \mathrm{L}$, normal $=136-145 \mathrm{mEq} / \mathrm{L}$ ). The patient's anticoagulation profile was within the therapeutic range $(\mathrm{INR}=1.4)$, and the platelet count was normal. Oxygen saturation on room air was normal. The patient's medication list included warfarin, digoxin, aspirin, metoprolol, montelukast, and atorvastatin.

Frontal chest radiography (Figure 1) was performed.

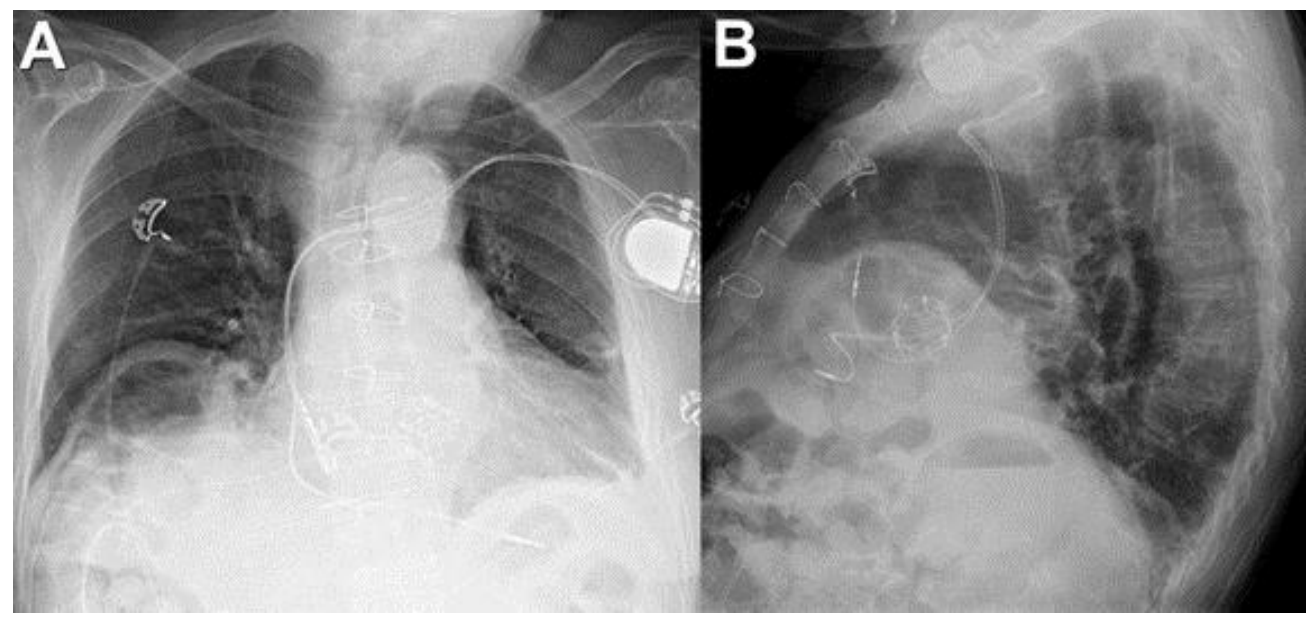

Figure 1. Frontal $(A)$ and lateral $(B)$ chest radiography.

Which of the following statements regarding the chest radiograph is most accurate?

1. Frontal chest radiography shows a cavitary lung mass

2. Frontal chest radiography shows focal consolidation suggesting aspiration pneumonia

3. Frontal chest radiography shows increased pressure edema

4. Frontal chest radiography shows malposition of the patient's left subclavian pacemaker

5. Frontal chest radiography shows rib fractures 


\section{Correct! \\ 5. Frontal chest radiography shows rib fractures}

The frontal and lateral chest radiographs show mildly displaced left postero-lateral rib fractures associated with soft tissue along the internal aspect of the infero-lateral left chest wall, consistent with pleural effusion or thickening, or possibly extrapleural hematoma, given the rib fractures (Figure 2).

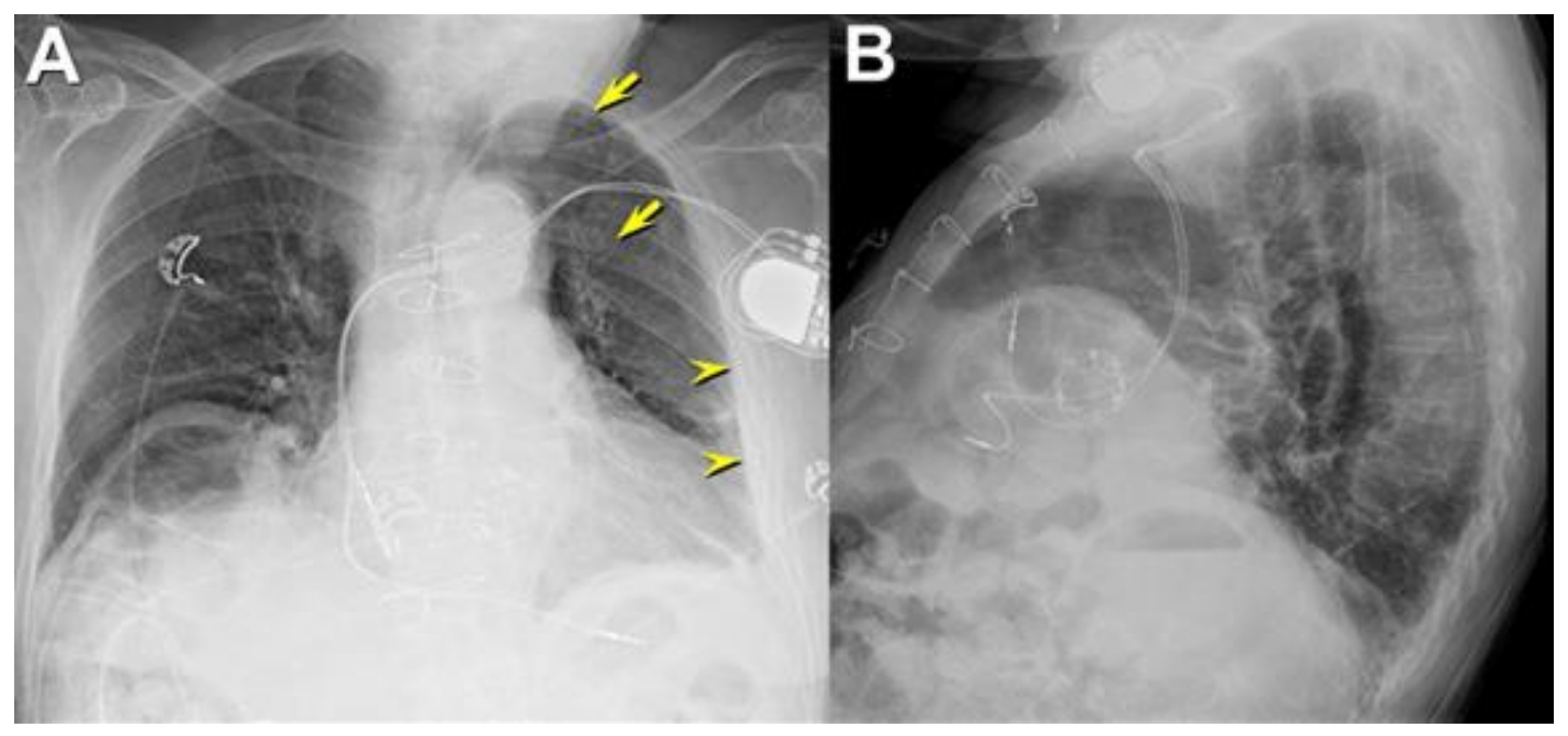

Figure 2. Frontal $(A)$ and lateral $(B)$ chest radiography shows mild cardiomegaly associated with an elevated right diaphragm with a left subclavian pacemaker with leads projected in the right atrial appendage and right ventricle as well as transcatheter aortic valve implantation (Edwards-Sapien, \#26). The patient is rotated towards his left (right

anterior oblique). A small amount of soft tissue along the left chest wall, which may reflect pleural effusion or pleural thickening (arrowheads), is present. Left postero-lateral rib fractures are seen (arrows).

There is some basal linear opacity consistent with atelectasis, but no focal consolidation to suggest pneumonia, either community-acquired or aspiration-related, is seen, and no cavitary lung mass is evident. No interlobular septal thickening to suggest increased pressure edema is present. The left subclavian pacemaker leads appropriately project in the right atrial appendage and right ventricle, and the transcatheter aortic valve implant appears normally positioned.

Further questioning of the patient's relatives indicated that he was taking opiates for chest pain related to a left chest wall injury that occurred 15 days earlier, diagnosed at an outside facility, after the patient fell on a rock. It was thought that the patient's altered mental status may the result of his electrolyte abnormalities in combination with his opiate use. His digoxin level was found to be elevated $(3.4 \mathrm{ng} / \mathrm{mL}$, normal $=0.8-2$ $\mathrm{ng} / \mathrm{mL}$, toxic $>2.5 \mathrm{ng} / \mathrm{mL}$ ), but this level was also trended downward after doses were withheld. Head CT was performed, which showed no acute abnormalities. On the 
second day of his admission, while his delirium persisted, his serum sodium had improved $(132 \mathrm{mEq} / \mathrm{L}$; normal $=136-145 \mathrm{mEq} / \mathrm{L})$ and his white blood cell count decreased as well. Echocardiography showed a left ventricular ejection fraction of $54 \%$ with normal right ventricular function. The aortic valve prosthesis appeared normal positioned with an aortic valve gradient of $6 \mathrm{mmHg}$.

A blood culture was positive for Streptococcus, thought to reflect bacteremia from the urinary tract infection and previous episodes of self-catheterization. A urinalysis suggested possible urinary tract infection, for which ceftriaxone therapy was begun, and later penicillin was added.

The possibility of infective endocarditis was considered, for which transesophageal echocardiography was performed. This examination showed normal biventricular function, the aortic valve prosthesis appeared normal positioned with an aortic valve gradient of $4 \mathrm{mmHg}$, no pericardial effusion was seen, and no valvular vegetations were present. After this procedure, the patient was noted to be hypotensive (systolic blood pressure $=65 \mathrm{mmHg}$ ) and tachycardic, but afebrile; he was treated with intubation and aggressive fluid resuscitation and transfusion was begun. Frontal chest radiography (Figure 3) was performed.

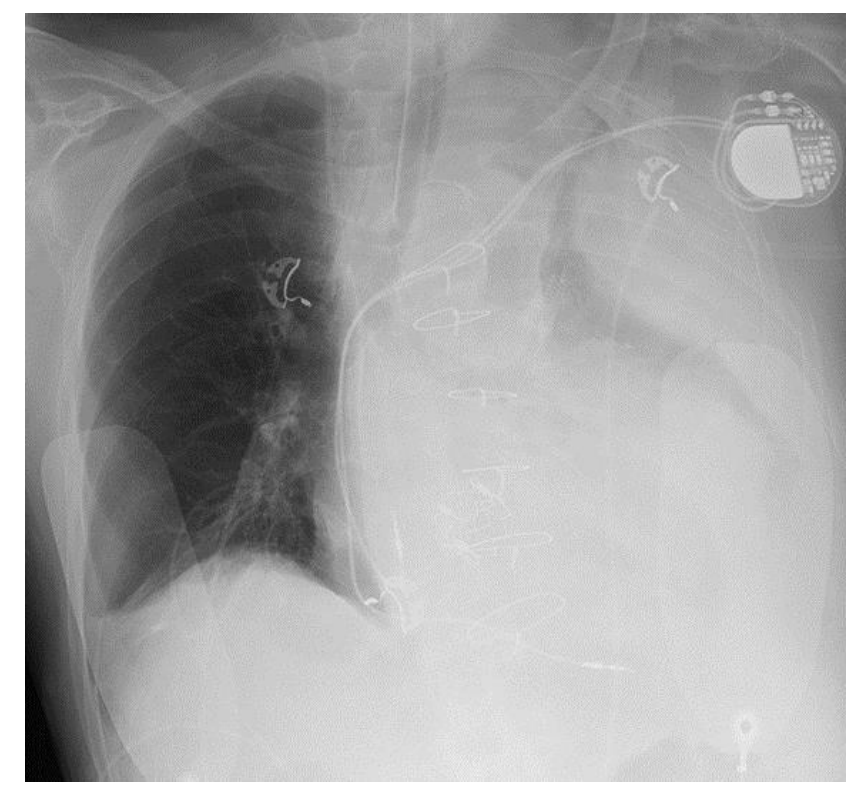

Figure 3. Frontal chest radiography.

Which of the following statements regarding the chest radiograph is most accurate?

1. The frontal chest radiograph shows a large pleural effusion

2. The frontal chest radiograph shows embolization of the aortic valve prosthesis

3. The frontal chest radiograph shows extravascular migration of the pacemaker

4. The frontal chest radiograph shows marked worsening of cardiomegaly

5. The frontal chest radiograph shows new pulmonary arterial enlargement 


\section{Correct!}

\section{The frontal chest radiograph shows a large pleural effusion}

The frontal chest radiograph shows near-complete opacification of the left thorax, representing a large left pleural effusion. No clear evidence of new or worsening cardiomegaly is noted, but detecting such would be difficult given the obscuration of the left cardiac border. The pacemaker leads and aortic valve prosthesis are in normal position and appear unchanged. The left pulmonary artery cannot be seen, but the right interlobar pulmonary artery is visualized and appears normal in size.

The patient underwent emergent repeat echocardiography at the bedside, which was limited, but showed a small left ventricular cavity with hyperdynamic function with a small right ventricular cavity with new mild-to-moderate right ventricular dysfunction, with a normal position of the interventricular septum. Only a tiny posterior pericardial effusion was seen. Left pleural effusion was visualized.

Which of the following possibilities is most likely to account for the patient's current condition?

1. Cardiac tamponade

2. Cardiogenic shock due to myocardial infarction from coronary artery vegetation embolization

3. Cardiogenic shock from right ventricular strain due to acute pulmonary embolism

4. Hemothorax due to intercostal artery laceration

5. Sepsis / systemic inflammatory response due to urinary tract infection 


\section{Correct! \\ 4. Hemothorax due to intercostal artery laceration}

Given the patients recent traumatic chest injury with rib fractures and the fact that he is anticoagulated, and new large left pleural effusion in a patient with hemorrhagic shock most likely is the result of intercostal artery laceration producing a large hemothorax. Sepsis is a consideration given the evidence of urinary traction infection and clinical suspicion for infective endocarditis, but the patient was afebrile, his decompensation was sudden, and his infection had been well-controlled. Cardiac tamponade could produce his hypotension, but would not explain the new large left pleural effusion, and the repeat bedside echocardiogram showed only a tiny pericardial effusion. Cardiogenic shock due to myocardial infarction merits consideration, but the patient's left ventricular function was normal-to-hyperdynamic, rather than showing regional wall motion abnormalities that typically result from myocardial ischemia. Furthermore, transesophageal echocardiography failed to reveal any vegetations. Finally, cardiogenic shock is a possibility give that the repeat bedside echocardiogram showed new moderate right ventricular systolic dysfunction and the patient's decompensation was acute; however, the pleural effusion is atypically large for effusions associated with thromboembolic disease and, while the echocardiogram showed new systolic dysfunction, it did not show leftward bowing of the interventricular septum or new right ventricular enlargement, both of which are typically encountered with large pulmonary emboli producing systemic hypotension.

Which of the following choices is most appropriate for the management of this patient?

1. ${ }^{99 m} \mathrm{Tc}$ ventilation-perfusion scintigraphy

2. Cardiac MRI

3. Contrast-enhanced thoracic CT

4. Repeat chest radiography with left lateral decubitus position

5. Thoracentesis 


\section{Correct! \\ 3. Contrast-enhanced thoracic CT}

Among the choices listed, contrast-enhanced CT would be most useful for confirming the working diagnosis of left hemothorax due to intercostal artery laceration resulting from left rib fractures. Lateral decubitus chest radiography is useful for confirming the presence of small pleural effusions and assessing whether or not such effusions are amenable to percutaneous drainage (when the decubitus image is performed with the affected thorax in a dependent position) or detection of pneumothorax (when the decubitus image is performed with the affected thorax in a non-dependent position). However, there is no uncertainty regarding the presence or absence of left pleural effusion in this patient. ${ }^{99 \mathrm{~m} T c-v e n t i l a t i o n-p e r f u s i o n ~ s c i n t i g r a p h y ~ i s ~ c o m m o n l y ~ u s e d ~ t o ~}$ assess for suspected acute or chronic thromboembolic disease or to assess for differential lung perfusion prior to thoracic surgery, but thromboembolic disease is not a likely cause for the patient's acute deterioration, and ${ }^{99 m T c-v e n t i l a t i o n-p e r f u s i o n ~}$ scintigraphy would not be a good test choice for such an acutely ill patient. Similarly, cardiac MR is not a suitable test for acutely ill patients, and probably would not provide any additional information not already known through recent echocardiography. Thoracentesis is commonly employed to sample or drain pleural effusions, but in a critically ill patient with presumed hemothorax, merely removing the left pleural liquid would not address the potential bleeding; if anything, such removal could reduce any pressure-related hemostatic effects the large hemothorax is providing. Finally, when pleural drainage of a large hemothorax is performed, it is typically conducted through placement of a large-bore surgically-placed thoracostomy tube, not bedside small-bore temporary catheter drainage.

A left-sided thoracostomy tube was placed by cardiothoracic surgery and the patient underwent thoracic CT (Figure 4).

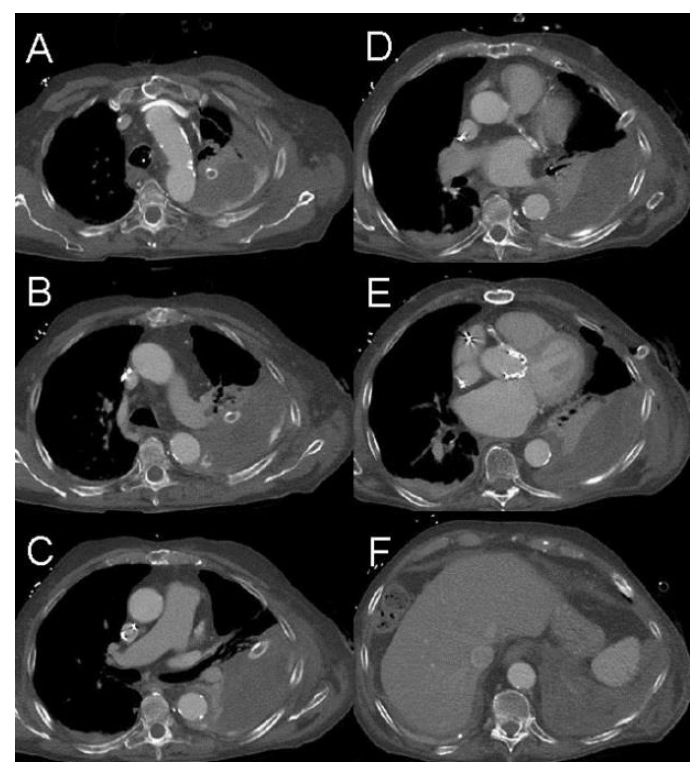

Figure 4. Axial contrast-enhanced thoracic CT in soft-tissue windows. 
Which of the following is the most accurate description of this this patient's CT findings?

1. The enhanced thoracic CT shows a left hydropneumothorax

2. The enhanced thoracic CT shows a left ventricular aneurysm

3. The enhanced thoracic CT shows a pulmonary artery aneurysm

4. The enhanced thoracic CT shows active bleeding in the left pleural space

5. The enhanced thoracic CT shows an aortic injury 


\section{Correct! \\ 4. The enhanced thoracic CT shows active bleeding in the left pleural space}

The enhanced thoracic CT shows hyperattenuation paralleling the internal aspect of the left thorax, which represents active extravasation of contrast-enhanced blood from an injured intercostal artery (Figure 5).

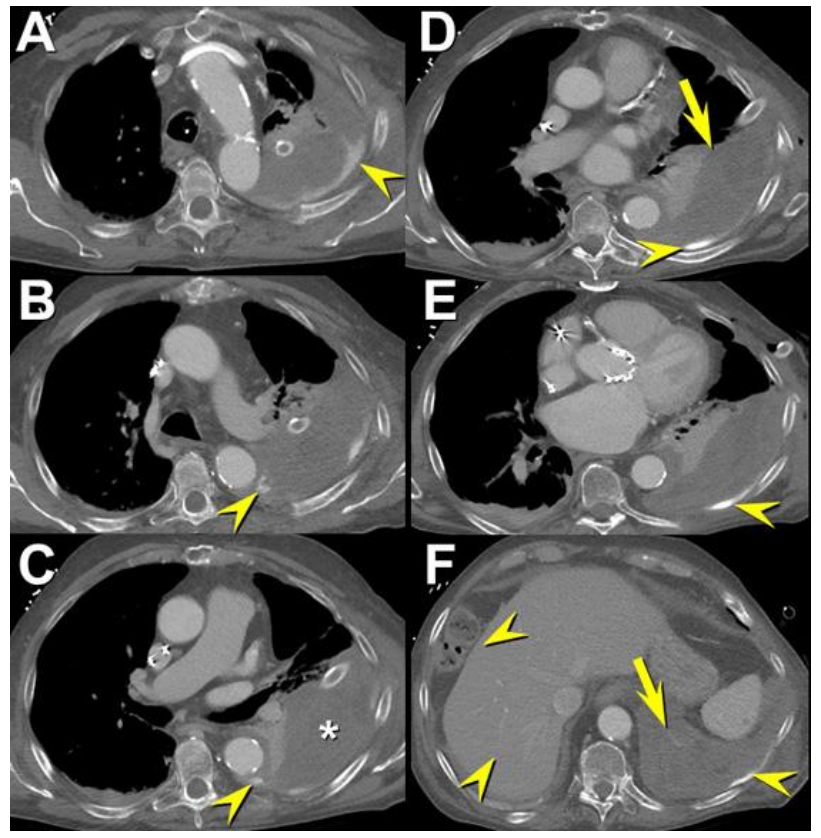

Figure 5. Axial contrast-enhanced thoracic CT shows a large left pleural effusion ( $\left.{ }^{*}\right)$. A thoracostomy tube is present in the left pleural space. There is linear hyperattenuation (arrowheads) in the peripheral left thorax, representing active contrast-enhanced blood extravasating from an injured intercostal artery. More inferiorly in the left pleural space, the pleural liquid appears mildly hyperattenuating and organized, consistent with retracting blood clot.

The heart appears normal, aside from coronary artery calcification, and no pulmonary arterial aneurysm is present. A large left pleural effusion is present, but there is no visible gas in the left pleural space, so no hydropneumothorax is present. The thoracic aorta shows atherosclerosis, but there is no evidence to suggest aortic injury.

Given the CT findings, which of the following choices is most appropriate for the management of this patient?

1. Continual thoracostomy tube drainage with close observation

2. Rigid bronchoscopy with airway stent placement

3. Catheter pulmonary angiography and embolization

4. Catheter aortography with intercostal artery selection and embolization

5. Repeated thoracentesis as needed 


\section{Correct! \\ 4. Catheter aortography with intercostal artery selection and embolization}

Active bleeding, as demonstrated on the contrast-enhanced thoracic CT, is an emergent situation requiring definitive therapy combined with supportive therapy (e.g., reversal of anticoagulant influences, fluid resuscitation, transfusion, etc.). In this context, definitive therapy could consist of immediate surgery for control of hemorrhage (this option was not offered in the choices above) or aortography with intercostal artery selection to identify the site of bleeding, followed by embolotherapy. Repeated thoracentesis may be a management option for chronic pleural effusions unassociated with hemodynamic compromise, such is chronic, recurrent malignant pleural effusions, but would not play a role here. Catheter pulmonary angiography and embolotherapy would not play a role here because the bleeding is not originating from the pulmonary arterial circulation; rather, the origin of the bleeding in the context of hemothorax is the intercostal arteries, and hence from the aorta. This patient's bleeding is not originating from the airways or bronchial circulation, so endoscopic therapy with rigid bronchoscopy and stent placement would not be an appropriate choice.

Further clinical course: The patient underwent catheter aortography with selective intercostal arteriography (Figure 6).

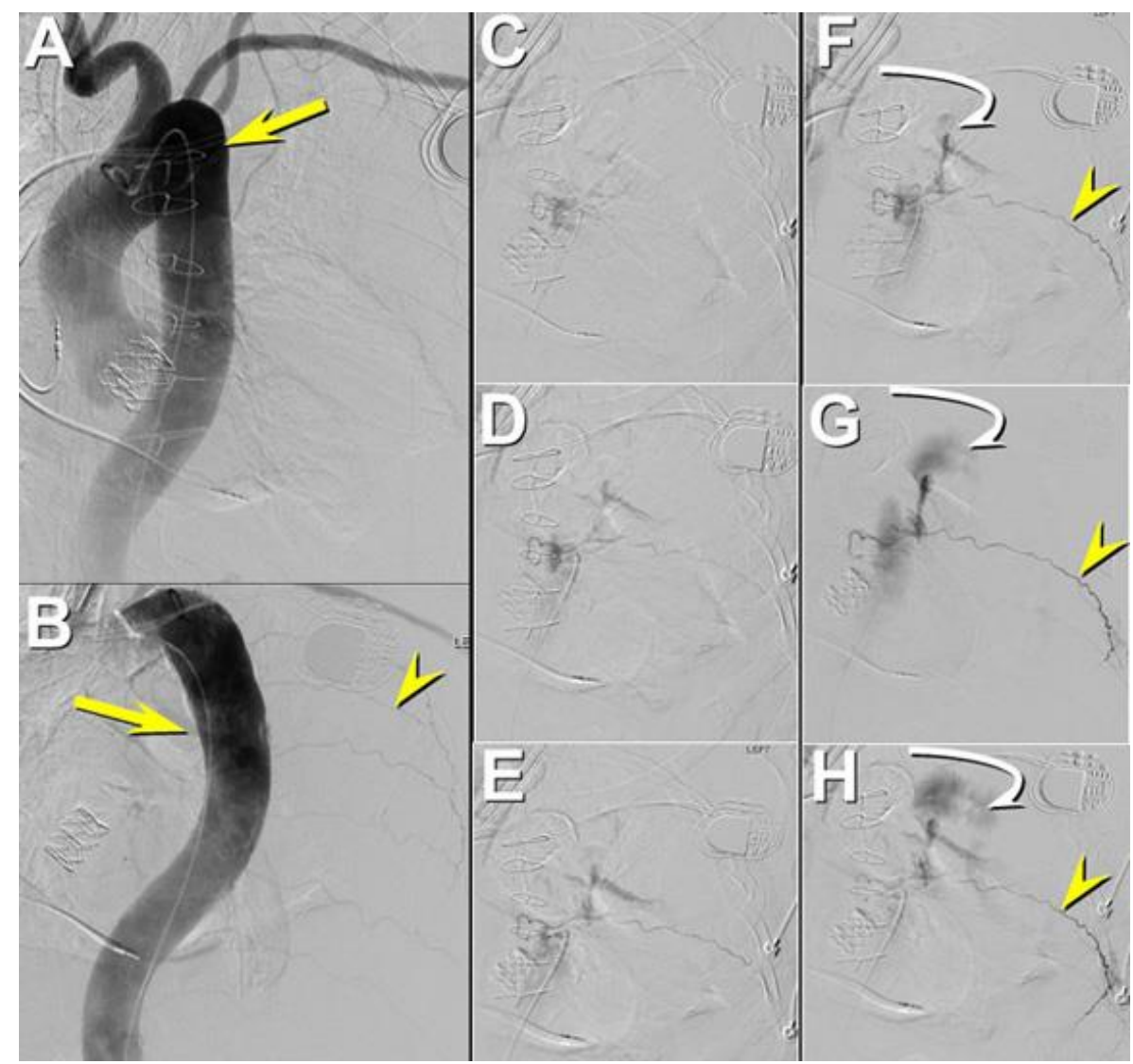

Figure 6. Catheter aortography (A and B) with selective intercostal arteriography $(C-H)$. 
Which of the following is the most accurate description of this this patient's aortography and intercostal arteriography findings?

1. Catheter aortography / intercostal angiography shows active contrast extravasation from an injured intercostal artery

2. Catheter aortography / intercostal angiography shows aortic rupture

3. Catheter aortography / intercostal angiography shows bronchial artery hypertrophy

4. Catheter aortography / intercostal angiography shows multiple intercostal arterial pseudoaneurysms

5. Catheter aortography / intercostal angiography shows no findings to suggest intercostal arterial injury 


\section{Correct! \\ 1. Catheter aortography / intercostal angiography shows active contrast extravasation from an injured intercostal artery}

The catheter aortography / intercostal arteriogram shows a progressive accumulation of amorphous contrast material as the imaging progresses from the early injection phase into the delayed phase, characteristic of contrast extravasation / active bleeding (Figure 7).

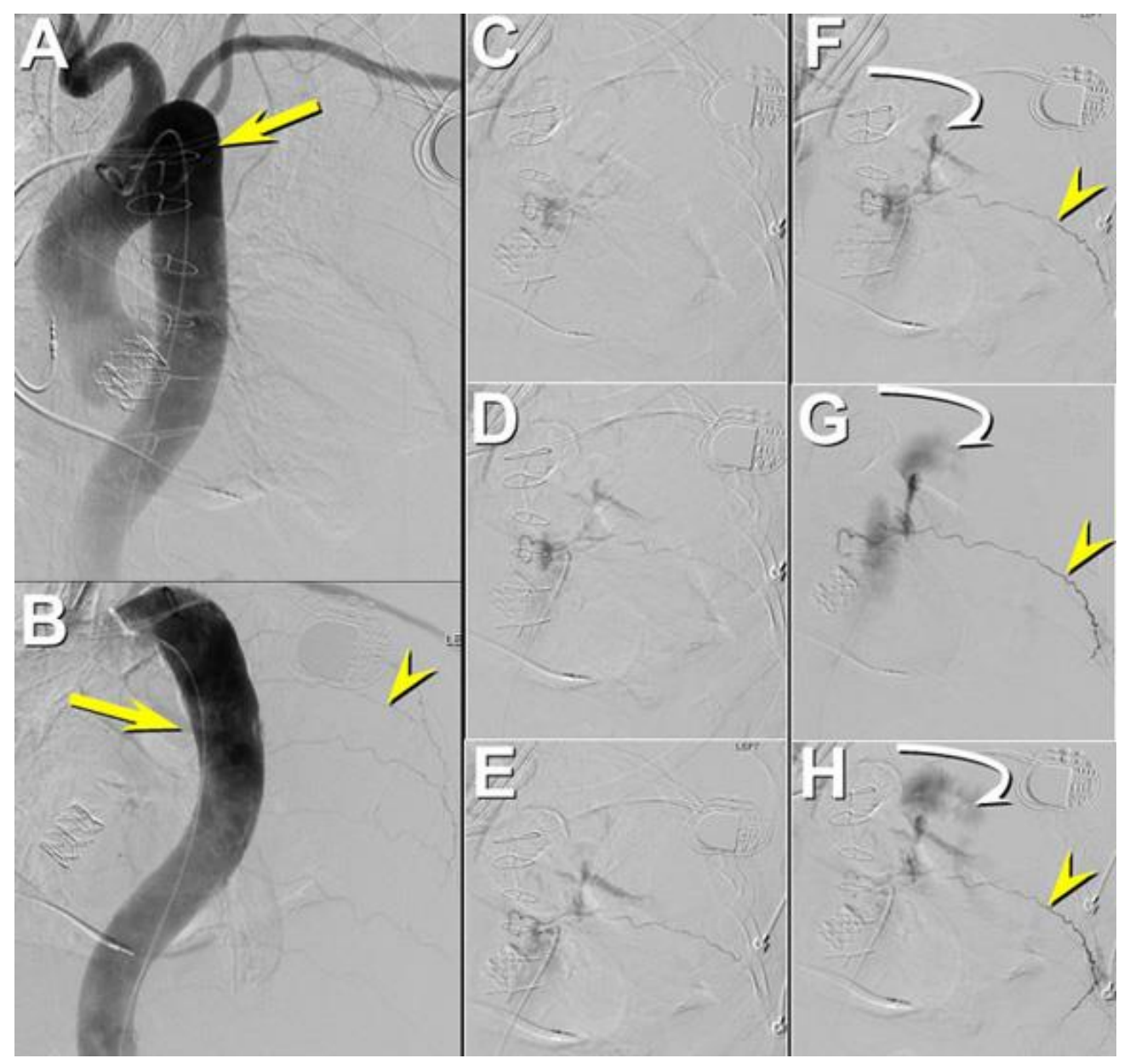

Figure 7. Catheter aortography (A and $B$ ) with selective intercostal arteriography $(C-D)$ shows a normal contour and caliber of the thoracic aorta; no evidence o of injury or pseudoaneurysm is present. The intercostal arteries (arrow head, B) are faintly visible on this aortic (i.e. non-selective) injection. With selection of the intercostal arteries, intercostal arterial opacification is improved (arrowheads, F-H). Images $\mathrm{C}-\mathrm{H}$ reflect progression of the

injection from earlier to delayed phases. As the imaging progresses, an amorphous contrast collection (curved arrows) develops and grows more pronounced as the intercostal arterial opacification fades, father into the delayed phase of the injection; the appearance is

typical of active contrast extravasation due to active bleeding. No intercostal arterial pseudoaneurysm or bronchial arterial hypertrophy is present (the latter were not selectively injected). 
The aorta appears normal as do the great vessels. The bronchial arteries are not well demonstrated on this examination as they are difficult to visualize with a non-selective aortic injection and they were not selectively cannulated for this study. No discrete, localized dilations of the intercostal arteries- or pseudoaneurysms- are evident.

The patient was treated with fluid resuscitation, vasopressor support, intubation with mechanical ventilation, and subsequently underwent video-assisted thoracoscopic surgery, which disclosed a large left hemothorax. The hemothorax was evacuated with placement of several large-bore thoracostomy tubes. Visual inspection of the aorta showed no evidence of injury. The left-sided rib fractures created a flail segment, and plate fixation and stabilization of the injured chest wall segment was being considered if the patient failed to wean from mechanical ventilation.

Diagnosis: Hemothorax with active bleeding, due to rib fractures

\section{References}

1. Chapman BC, Overbey DM, Tesfalidet F, Schramm K, Stovall RT, French A, Johnson JL, Burlew CC, Barnett C, Moore EE, Pieracci FM. Clinical utility of chest computed tomography in patients with rib fractures: CT Chest and Rib Fractures. Arch Trauma Res. 2016 Sep 13;5(4):e37070. [CrossRef][PubMed]

2. Bugaev N, Breeze JL, Alhazmi M, Anbari HS, Arabian SS, Rabinovici R. Displacement Patterns of Blunt Rib Fractures and Their Relationship to thoracic coinjuries: minimal displacements count. Am Surg. 2016;82(3):199-206. [PubMed]

3. Masuda R, Ikoma Y, Oiwa K, Nakazato K, Takeichi H, Iwazaki M. Delayed hemothorax superimposed on extrapleural hematoma after blunt chest injury: a case report. Tokai J Exp Clin Med. 2013;38(3):97-102. [PubMed]

4. Yoneyama H, Arahata M, Temaru R, Ishizaka S, Minami S. Evaluation of the risk of intercostal artery laceration during thoracentesis in elderly patients by using 3D-CT angiography. Intern Med. 2010;49(4):289-92. [CrossRef] [PubMed]

5. Chemelli AP, Thauerer M, Wiedermann F, Strasak A, Klocker J, ChemelliSteingruber IE. Transcatheter arterial embolization for the management of iatrogenic and blunt traumatic intercostal artery injuries. J Vasc Surg. 2009;49(6):1505-13. [CrossRef] [PubMed]

6. Hagiwara A, Iwamoto S. Usefulness of transcatheter arterial embolization for intercostal arterial bleeding in a patient with burst fractures of the thoracic vertebrae. Emerg Radiol. 2009;16(6):489-91. [CrossRef] [PubMed] 